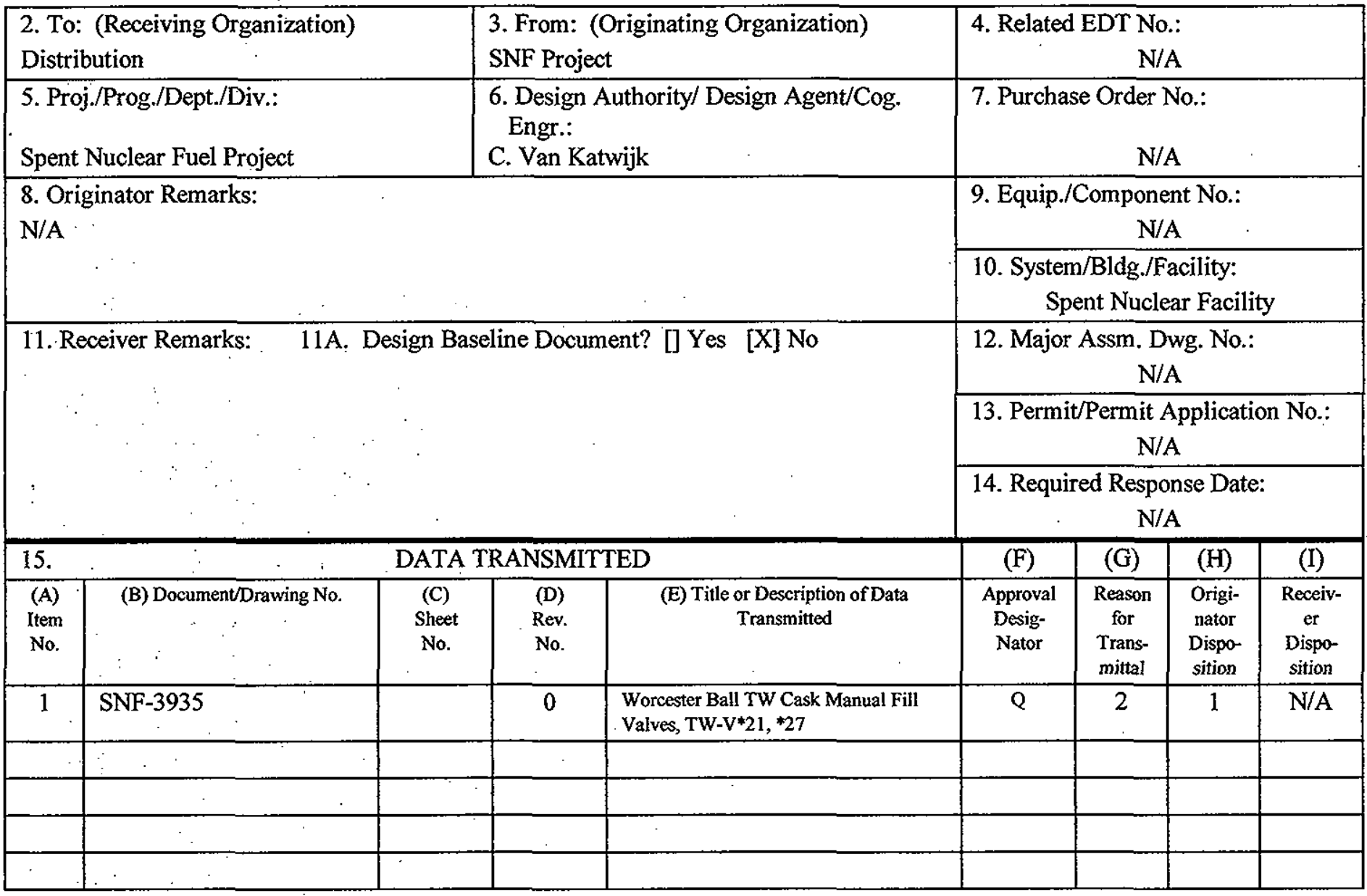

16.

KEY

\begin{tabular}{|c|c|c|c|c|}
\hline Approval Designator (F) & & Reason for Transmittal (G) & \multicolumn{2}{|c|}{ Disposition (H) \& (I) } \\
\hline $\begin{array}{l}E, S, Q, D \text { or N/A } \\
\text { (see WHC-CM-3-5, } \\
\text { Sec.12.7) }\end{array}$ & $\begin{array}{l}\text { 1. Approval } \\
\text { 2. Release } \\
\text { 3. Information }\end{array}$ & $\begin{array}{l}\text { 4. Review } \\
\text { 5. Post-Review } \\
\text { 6. Dist. (Receipt Acknow. Required) }\end{array}$ & $\begin{array}{l}\text { 1. Approved } \\
\text { 2. Approved w/comment } \\
\text { 3. Disapproved w/comment }\end{array}$ & $\begin{array}{l}\text { 4. Reviewed no/comment } \\
\text { 5. Reviewed w/comment } \\
\text { 6. Receipt acknowledged }\end{array}$ \\
\hline
\end{tabular}
17. SIGNATURE/DISTRIBUTION

(See Approval Designator for required signatures)

\begin{tabular}{|c|c|c|c|c|c|c|}
\hline $\begin{array}{l}\text { (G) } \\
\text { Rea- } \\
\text { son }\end{array}$ & $\begin{array}{l}\text { (H) } \\
\text { Disp. }\end{array}$ & (J) Name (K) Signature (L) Date (M) MSIN & $\begin{array}{l}\text { (G) } \\
\text { Rea- } \\
\text { Son }\end{array}$ & $\begin{array}{l}\text { (H) } \\
\text { Disp. }\end{array}$ & (J) Name & (K) Signature (L) Date (M) MSIN \\
\hline 2 & 1 & Designated Engineer C. Van K & & & & \\
\hline 2 & 1 & Design Authority J. J. Inwin & & & & \\
\hline 2 & 1 & QA T. D. Hays $\square D$ A & & & & \\
\hline & & & & & & \\
\hline & & & & & & \\
\hline & & & & & & . \\
\hline
\end{tabular}

\begin{tabular}{|c|c|c|c|}
\hline Govan Katyislle & T. Chohor & s. & $\begin{array}{l}\text { 21. DOE APPROVAL (if required) } \\
\text { Ctrl. No. } \\
\text { D Approved } \\
\text { D Approved w/comments }\end{array}$ \\
\hline $\begin{array}{l}\text { Signature of ED Date } \\
\text { Originator }\end{array}$ & $\begin{array}{l}\text { Authorized Representative Date } \\
\text { for Receiving Organization }\end{array}$ & $\begin{array}{l}\text { Design Authority/ } \\
\text { Cognizant Manager }\end{array}$ & D Disapproved w/comments \\
\hline
\end{tabular}




\section{Worcester Ball TW Cask Manual Valves, TW-V*021, *027}

Carl Van Katwijk

Numatec Hanford Co, Richland, WA 99352

U.S. Department of Energy Contract DE-AC06-96RL13200

EDT/ECN: 626284

Org Code: $2 \mathrm{G} 300$

B\&R Code: 39EW40400
UC: 620

Charge Code: 105559/A000

Total Pages: 12

Key Words: Full port Ball valve

Abstract: Worcester Ball TW Cask Manual Fill Valves, TW-V*021, *027

CGI-SNF-D-47-P4-043

TRADEMARK DISCLAIMER. Reference herein to any specific commercial product, process, or service by trade name, trademark, manufacturer, or otherwise, does not necessarily constitute or imply its endorsement, recommendation, or favoring by the United States Government or any agency thereof or its contractors or subcontractors.

Printed in the United States of America. To obtain copies of this document, contact: Document Control Services, P.O. Box 950, Mailstop H6-08, Richland WA 99352, Phone (509) 372-2420; Fax (509) 376-4989.

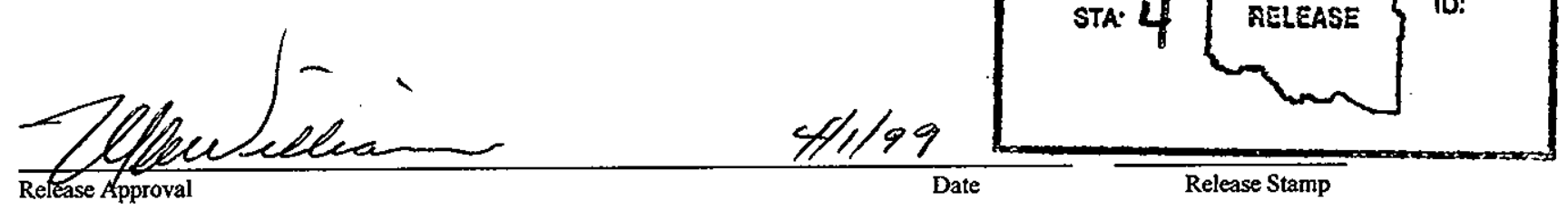


ECN No. NA CGINo. CGI-SNE-D-47-P4-043

Title: WORCESTER BALL TW CASK MANUAL FILL VALVES, TW-V $*_{021} * 027$

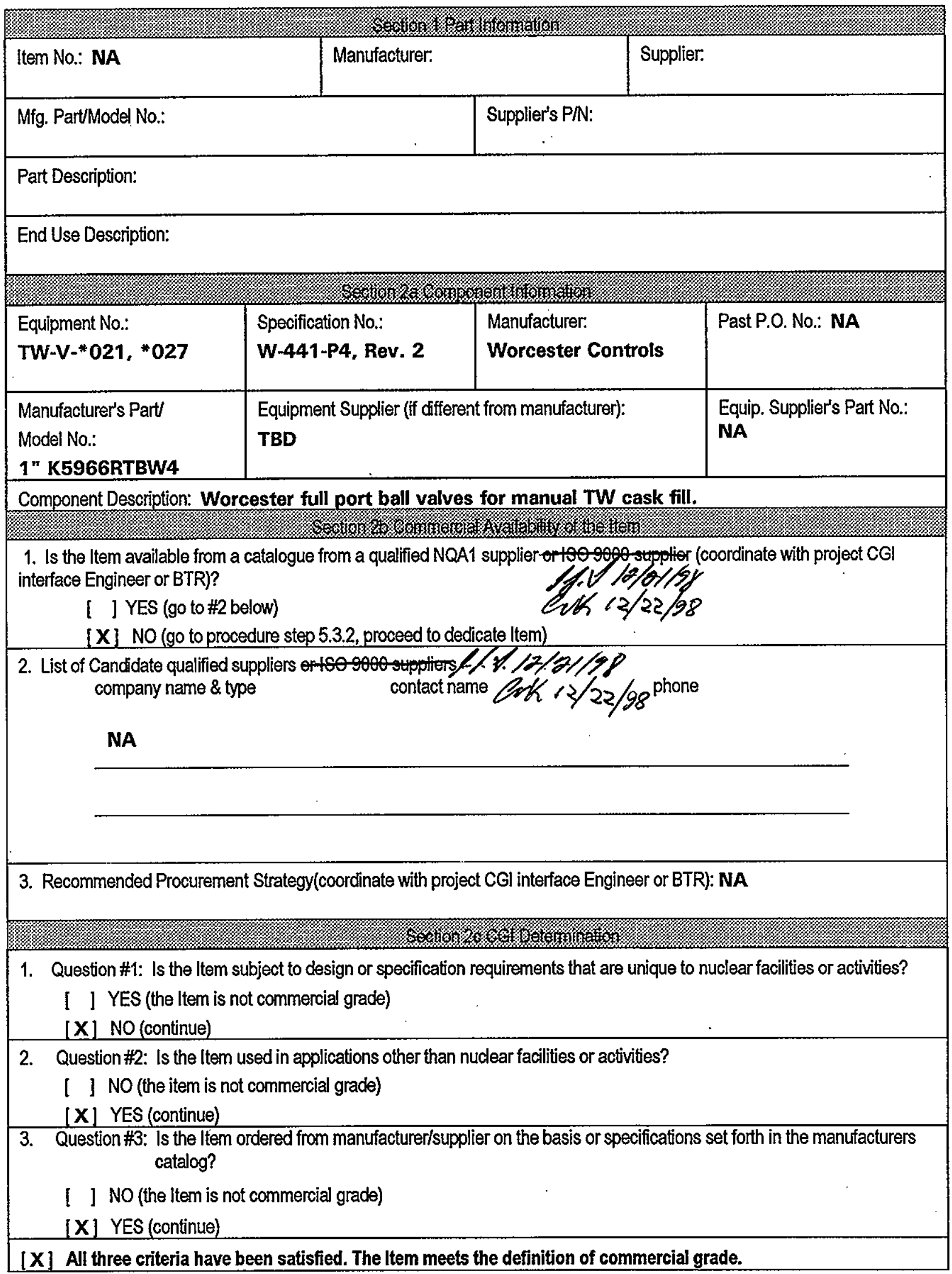


Commercial Grade Item Upgrade Dedication Form ECN No. NA CGINo. CGI-SNF-D-47-P4-043

Title: WORCESTER BALL TW CASK MANUAL FILL VALVES, TW-V *021, *027

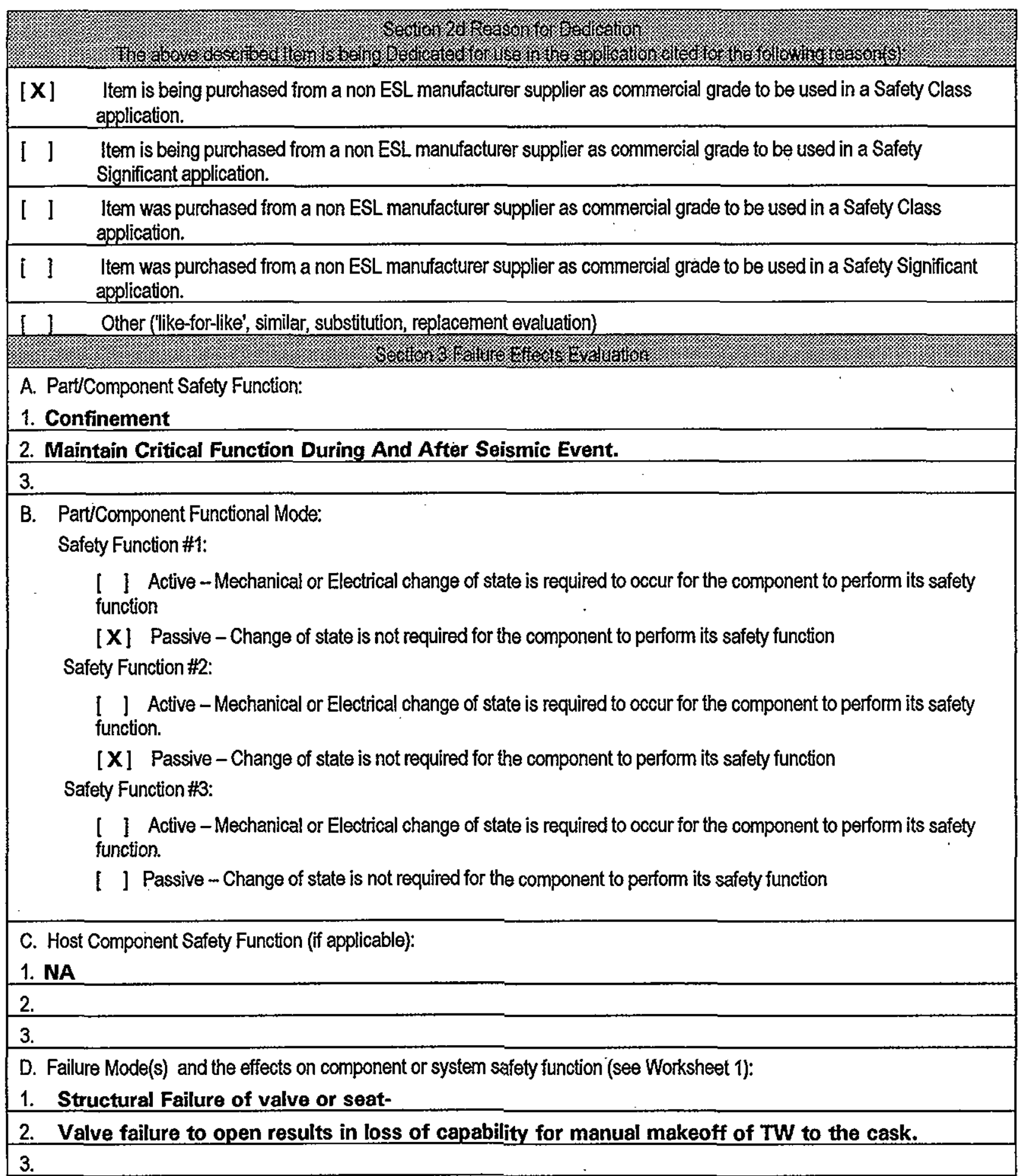




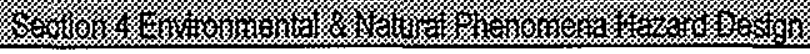

Environmental Qualification Required:

Yes [ I

No $[X]$

Environmental Condition B

Natural Phenomena Hazard (NPH) Design Required:

Yes $[\mathbf{X}]$

No [ ]

HNF-PRO-97, Rev. 0

WHE-OO-GW-OGS-3006

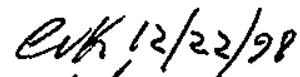

ellit $12 / 22 / 98$

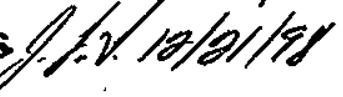

If yes: Environmental Qualification Requirements

Limiting Environmental Conditions:

Required Safety Functions:

Qualification Period:

If yes: NPH Design Requirements

Performance Category: PC-3

NPH Design Req'ts.: Seismic Condition A

Required Safety Functions: Confinement

-
[X] Safety Class (SC)
[ ] General Service
[ ] Safety Significant (SS)

if par/component classification is different from host component/system, document basis.

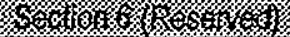

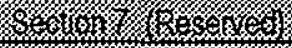

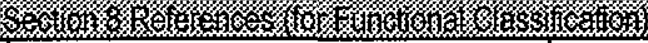

National Codes/Standards:

AMSE B31.1

B16 Series
Safety Analysis Report (SAR):

HNF-SD-SNF-SAR-002,

Rev. $4 A$
Drawings: H-1782403, Rev?

HNF-SD-SNF-SEL-002, Rev. 4

H $-1-82 / 61$, RCV $Z$

les $12 / 22 / 98$

$4 / 1.10 / 22 / 98$

Vendor Manual/Manufacturer/Supplier Information: Worcester Series 59 Full Port Ball Valve PB 451-22

Other: 
Commercial Grade Item Upgrade Dedication Form

ECN No. NA CGINo. CGI-SNF-D-47-P4-043

Title: WORCESTER BALL TW CASK MANUAL FILL VALVES, TW-V *021, *027
Rev. No. 0

Page 4 of 11

SNF 3935

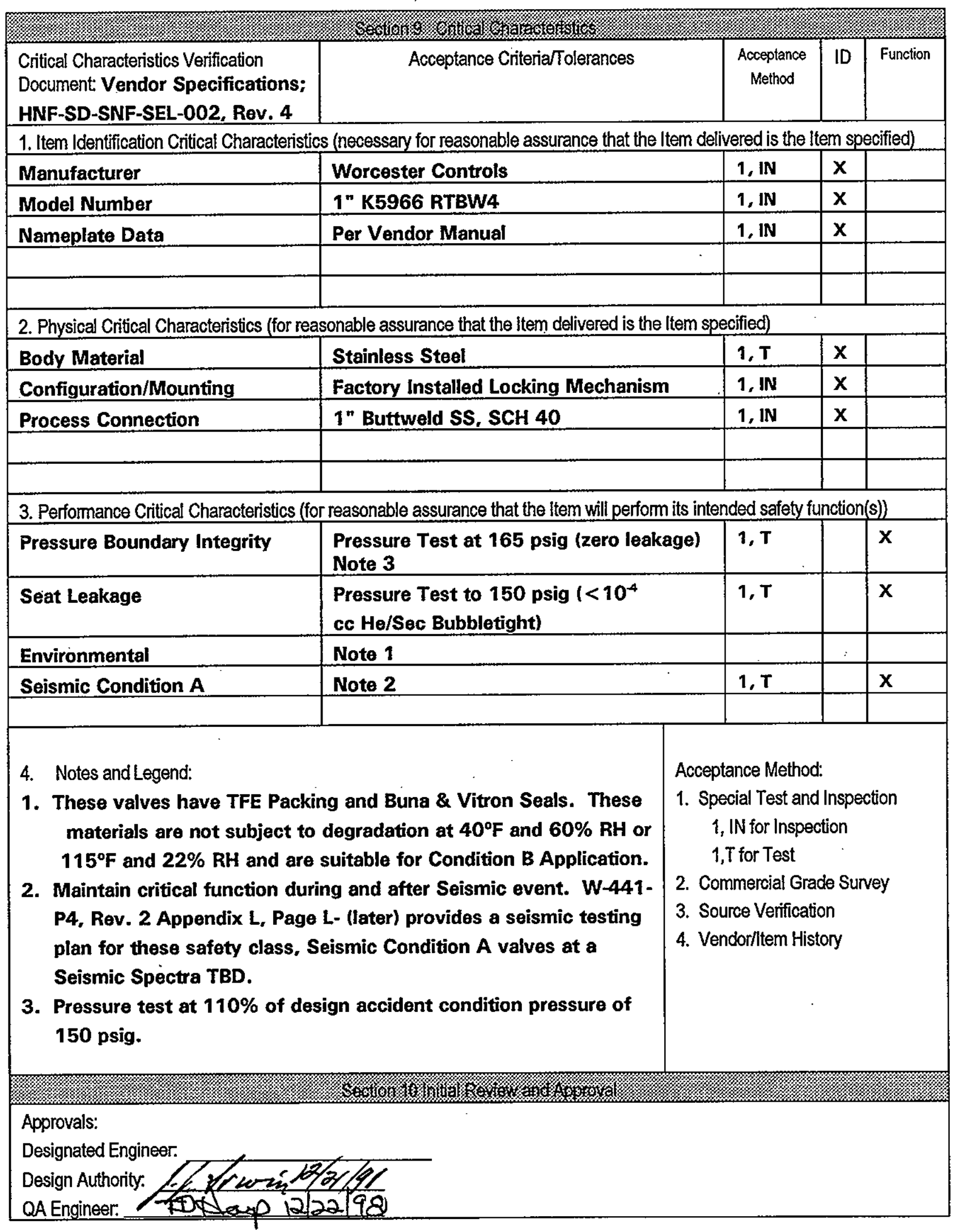


Title: WORCESTER BALL TW CASK MANUAL FLLL VALVES, TW-V *021, *027

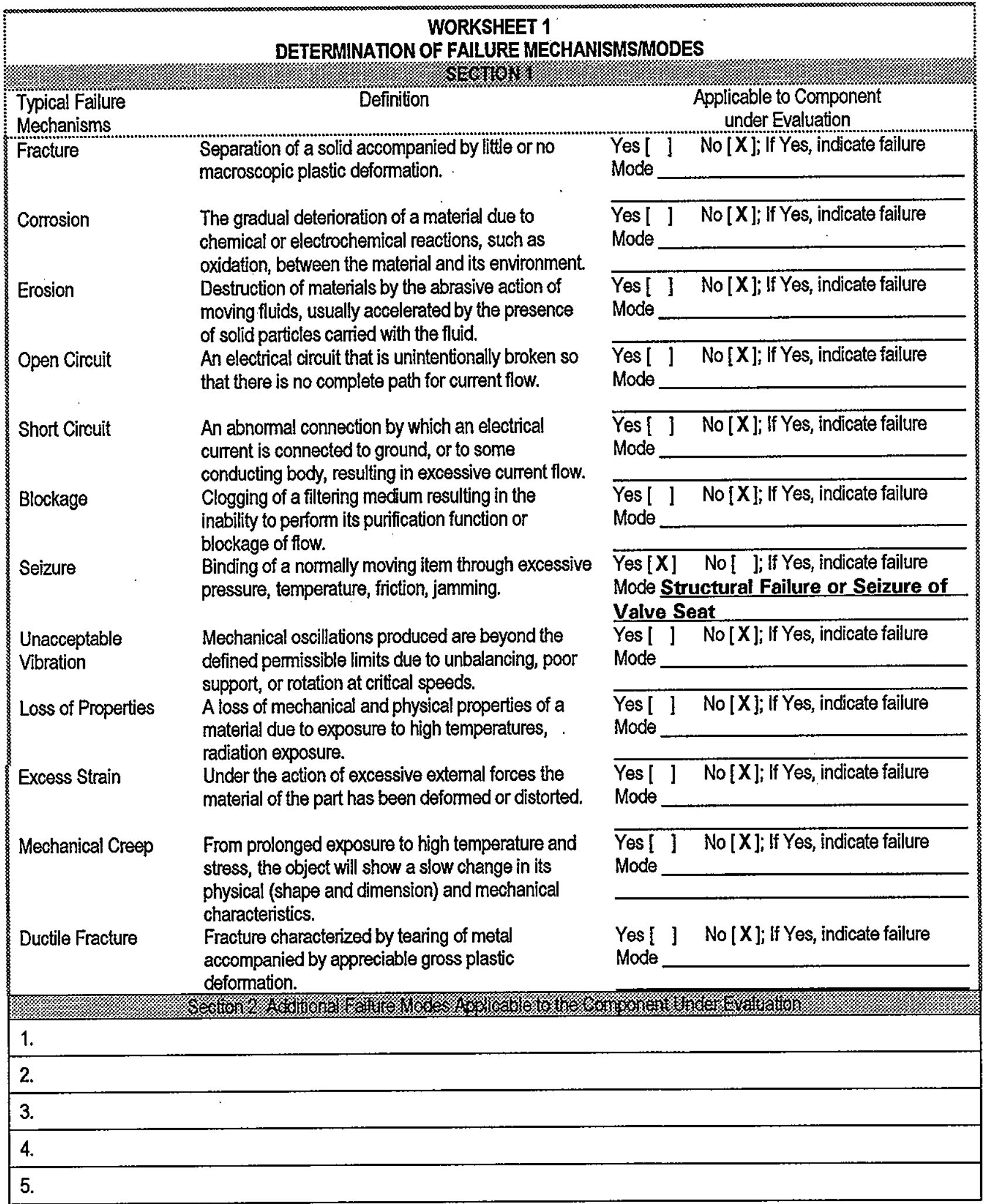


Commercial Grade Item Upgrade Dedication Form ECN No. NA CGINo. CGI-SNF-D-47-P4-043

Title: WORCESTER BALL TW CASK MANUAL FILL VALVES, TW-V *021, *027

\section{CHECKLIST 1}

\section{ACCEPTANCE METHOD 1.}

\section{SPECIAL TEST/INSPECTION VERITICATION}

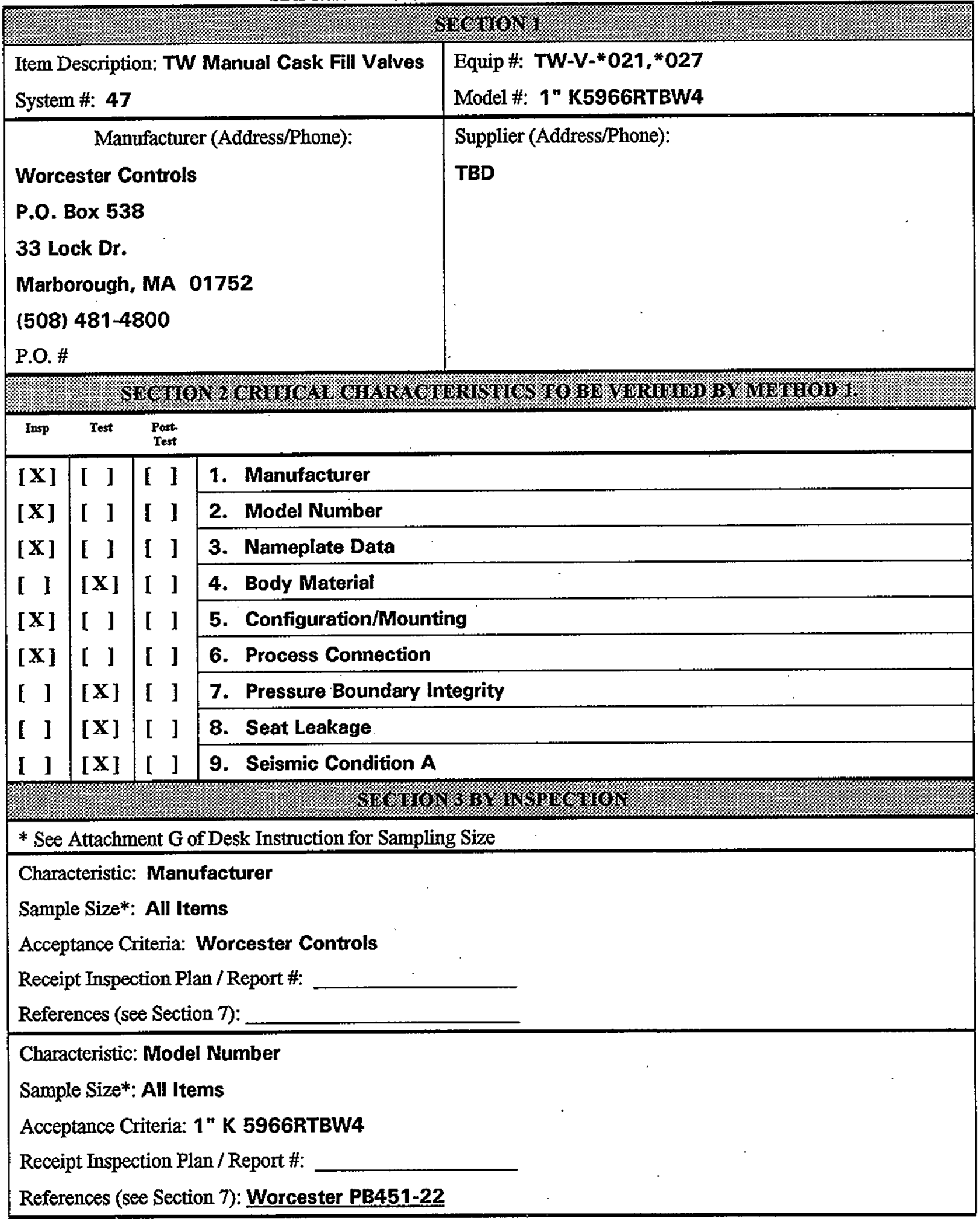


Commercial Grade Item Upgrade Dedication Form

ECN No. NA CGI No. CGI-SNF-D-47-P4-043

Titie: WORCESTER BALL TW CASK MANUAL FILL VALVES, TW-V

*021, *027

Rev. No. 0

Page 7 of 11

Characteristic: Nameplate Data

Sample Size*: All Items

Acceptance Criteria: Per Vendor Manual

Receipt Inspection Plan / Report \#:

References (see Section 7): Worcester PB451-22

Characteristic: Configuration/Mounting

Sample Size*: All Items

Acceptance Criteria: Factory Installed Locking Mechanism

Receipt Inspection Plan / Report \#:

References (see Section 7):

Characteristic: Process Connection

Sample Size*: All Items

Acceptance Criteria: 1" Buttweld SS, SCH 40

Receipt Inspection Plan / Report \#:

References (see Section 7):

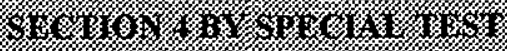

* See Attachment G of Desk Instruction for Sampling Size

\begin{tabular}{l|l}
\hline Test To Be Performed by: & Number of Items to be Tested:
\end{tabular}

[ ] Purchaser

[ ] Supplier/Manufacturer**

Test/Inspection Location:

[ ] Other

Characteristic for Test: Body Material

Acceptance Criteria: Stainless Steel

Sample Size*: Normal Sampling Size

Actual Test Value:

Test Plan and Report \#:

References (see Section 7):

Characteristic for Test: Pressure Boundary Integrity

Acceptance Criteria: Pressure Test at 165 psig (zero leakage)

Sample Size*: Normal Sampling Size

Actual Test Value:

Test Plan and Report \#:

References (see Section 7):

Characteristic for Test: Seat Leakage

Acceptance Criteria: Pressure Test to $150 \mathrm{psig}\left(<10^{4} \mathrm{cc} \mathrm{He} / \mathrm{Sec}\right.$ Bubbletight)

Sample Size*: Normal Sampling Size

Actual Test Value:

Test Plan and Report \#: References (see Section 7): 
Commercial Grade Item Upgrade Dedication Form Rev. No. 0

ECN No. NA CGINo. CGI-SNF-D-47-P4-043

Page 8 of 11

Title: WORCESTER BALL TW CASK MANUAL FILL VALVES, TW-V $\star_{021} *_{027}$

SNF 3935

Characteristic for Test: Seismic Condition A

Acceptance Criteria: Maintain Critical Function During and After Seismic Event.

Sample Size*: Normal Sampling Size

Actual Test Value:

Test Plan and Report \#: References (see Section 7):

**If Supplier/Manufacturer or Other, Refer to CGI Checklist-2 for Support Information 
Commercial Grade Item Upgrade Dedication Form

ECN No. NA CGI No. CGI-SNF-D-47-P4-043

Title: WORCESTER BALL TW CASK MANUAL FILL VALVES, TW-V *021, *027

Page 9 of 11
Sinf 3935

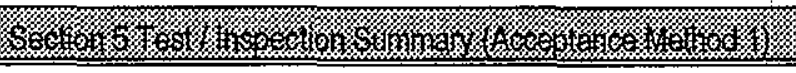

\section{SUMMARY OF VERIFUED CRITICAL CHARACTERISTICS, THEUR VERIFICATION METHODS, AND RESULTS}

\section{ITEM DESCRIPTION:}

\begin{tabular}{|c|c|c|c|c|c|c|c|c|c|c|c|}
\hline \multicolumn{4}{|c|}{ Critical Characteristics } & \multicolumn{8}{|c|}{ Verification Results } \\
\hline Critical Characteristics & Acceptance Criteria/Tolerances & $\mathbb{D}$ & Function & $\begin{array}{c}\text { Method } \\
\text { T/N }\end{array}$ & $\begin{array}{c}\text { Procedure } \\
\text { or RR\# }\end{array}$ & $\begin{array}{l}\text { Check- } \\
\text { list ID. }\end{array}$ & $\begin{array}{c}\text { Number } \\
\text { Tested }\end{array}$ & $\begin{array}{c}\text { Number } \\
\text { Failed }\end{array}$ & $\begin{array}{c}\text { Verifying } \\
\text { Organization }\end{array}$ & Printed Name Signature & Date \\
\hline Manufacturer & Worcester Controls & $\mathbf{X}$ & & & & & & & & & \\
\hline Model Number & 1" K5966 RTBW4 & $\mathbf{x}$ & & & & & & & & & \\
\hline Nameplate Data & Per Vendor Manual & $\mathbf{x}$ & & & & & & & & & \\
\hline Body Material & Stainless Steel & $\mathbf{X}$ & & & & & & & & & \\
\hline Configuration/Mounting & $\begin{array}{l}\text { Factory Installed Locking } \\
\text { Mechanism. }\end{array}$ & $\mathbf{X}$ & & & & & & & & & \\
\hline Process Connection & $\begin{array}{l}\text { 1" Buttweld SS, SCH } \\
40\end{array}$ & $\mathbf{X}$ & & & & & & & & & \\
\hline $\begin{array}{l}\text { Pressure Boundary } \\
\text { Integrity }\end{array}$ & $\begin{array}{l}\text { Pressure Test at } 165 \\
\text { psig (zero leakage) }\end{array}$ & & $\mathbf{X}$ & & & & & & & & \\
\hline Seat Leakage & $\begin{array}{l}\text { Pressure Test to } 150 \\
\text { psig }\left(<10^{4} \text { cc } \mathrm{He} / \mathrm{Sec}\right. \\
\text { Bubbletight) }\end{array}$ & & $\mathbf{X}$ & & & & & & & & \\
\hline Seismic Condition A & $\begin{array}{l}\text { Maintain Critical } \\
\text { Function }\end{array}$ & & $\mathbf{X}$ & & & & & & & & \\
\hline
\end{tabular}


Commercial Grade Item Upgrade Dedication Form

ECN No. NA CGINo. CGI-SNF-D-47-P4-043

Title: WORCESTER BALL TW CASK MANUAL FLL VALVES, TW-V *021, *027

\section{DISPOSITION OF UNVERIFIED OR FAILED CRITICAL CHARACTERISTICS}

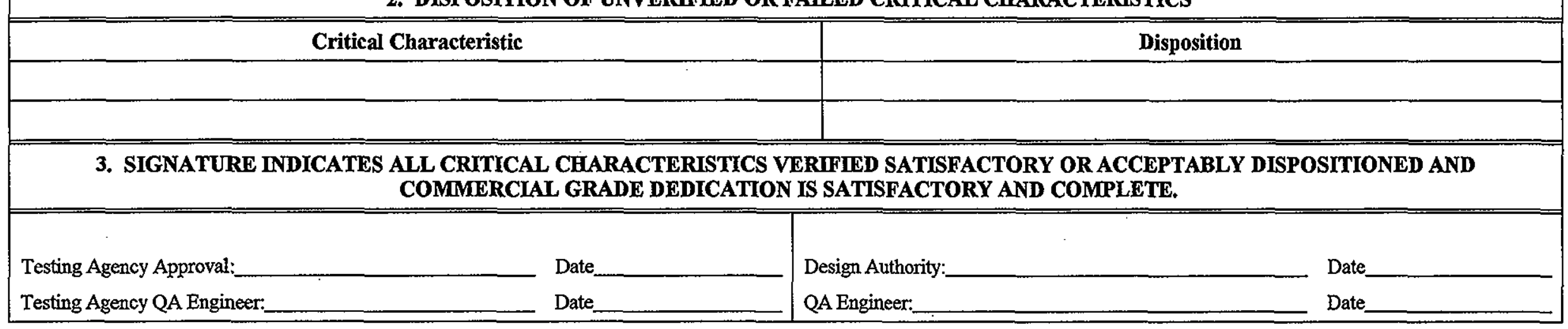


Commercial Grade Item Upgrade Dedication Form

ECNNo. NA CGI No. CGI-SNF-D-47-P4-043

Title: WORCESTER BALL TW CASK MANUAL FILL VALVES, TW-V *021, *027
Rev. No. 0

Page 11 of 11

SNF 3935

\begin{tabular}{|c|c|c|}
\hline \multicolumn{2}{|l|}{ Name } & Phone \\
\hline \multicolumn{2}{|l|}{ Design Authority } & ) \\
\hline \multicolumn{2}{|l|}{ QA } & ) \\
\hline \multicolumn{2}{|l|}{ QC } & ) \\
\hline \multicolumn{2}{|l|}{ Cog - Engineer } & ) \\
\hline \multicolumn{2}{|l|}{ CGI Engineer } & ). \\
\hline \multicolumn{2}{|l|}{ Procurement Engineer } & ) \\
\hline \multicolumn{2}{|c|}{ 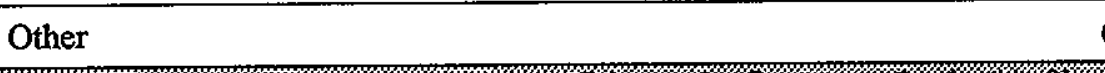 } & ) \\
\hline 4. & 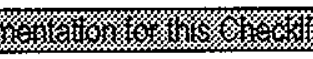 & \\
\hline \multicolumn{3}{|c|}{\begin{tabular}{|l|l} 
Initial Procurement Documents & For Critical Characteristics
\end{tabular}} \\
\hline \multicolumn{3}{|l|}{ [ ] Drawings: } \\
\hline \multicolumn{3}{|l|}{ [ ] Manuals (specify type \& number): } \\
\hline \multicolumn{3}{|l|}{ [ ] Design Calculations } \\
\hline \multicolumn{3}{|l|}{ [ ] Installation Instructions } \\
\hline \multicolumn{3}{|l|}{ [ ] Operation Instructions } \\
\hline \multicolumn{3}{|l|}{ [ ] Calibration Instructions } \\
\hline \multicolumn{3}{|l|}{ [ ] Manufacturer's Recommended Spare Parts List } \\
\hline \multicolumn{3}{|l|}{ [ ] Other: } \\
\hline \multicolumn{3}{|l|}{ Procurement Documents } \\
\hline \multicolumn{3}{|l|}{ [ ] Certificate of Conformance/Compliance } \\
\hline \multicolumn{3}{|l|}{ [ ] Seismic Qualification Certificate } \\
\hline \multicolumn{3}{|l|}{ [ ] Environmental Qualification Certificate } \\
\hline \multicolumn{3}{|l|}{ [ ] Test Report (s): } \\
\hline \multicolumn{3}{|l|}{ [ I Inspection Report (s): } \\
\hline \multicolumn{3}{|l|}{ [ ] CMTRs for ASME Pressure Retaining Materials } \\
\hline \multicolumn{3}{|l|}{ [ ] Valve Seat Leakage Report } \\
\hline \multicolumn{3}{|l|}{ [ ] Weld Records } \\
\hline \multicolumn{3}{|l|}{ [ ] Material Traceability Record } \\
\hline [ 1 Other: & & \\
\hline
\end{tabular}

